\title{
Self-Organization versus Watchmaker: ambiguity of molecular recognition and design charts of cellular circuitry
}

\author{
Alexei Kurakin \\ Buck Institute for Age Research, 8001 Redwood Blvd., Novato, CA 94945 \\ Tel. 415-8985750; Fax: 415-2092230; E-mail: ak@alexeikurakin.org
}

\begin{abstract}
A large body of experimental evidence indicates that the specific molecular interactions and/or chemical conversions depicted as links in the conventional diagrams of cellular signal transduction and metabolic pathways are inherently probabilistic, ambiguous, and context-dependent. Being the inevitable consequence of the dynamic nature of protein structure in solution, the ambiguity of protein-mediated interactions and conversions challenges the conceptual adequacy and practical usefulness of the mechanistic assumptions and inferences embodied in the design charts of cellular circuitry.

It is argued that the reconceptualization of molecular recognition and cellular organization within the emerging interpretational framework of self-organization, which is expanded here to include such concepts as bounded stochasticity, evolutionary memory, and adaptive plasticity, offers a significantly more adequate representation of experimental reality than conventional mechanistic conceptions do. Importantly, the expanded framework of self-organization appears to be universal and scale-invariant, providing conceptual continuity across multiple scales of biological organization, from molecules to societies. This new conceptualization of biological phenomena suggests that such attributes of intelligence as adaptive plasticity, decision-making, and memory are enforced by evolution at different scales of biological organization and may represent inherent properties of living matter.
\end{abstract}

Keywords: self-organization; adaptive plasticity; evolutionary memory; bounded stochasticity; molecular recognition; cellular organization

7 July 2007, Novato, CA

\section{Introduction}

The spectacular achievements of mechanical and electrical engineering sanctified the application of classico-mechanistic concepts and interpretations to the biological phenomena studied by life scientists at the molecular and cellular scales. From the beginning and for a long time the cell has been analyzed largely in the spirit and by the methods of reverse engineering. As a consequence, the complexity of the cell is perceived to be different from the complexity of modern aircrafts or computers only in terms of quantity but not quality. The mechanistic metaphors such as "genetic program", "protein engineering", "drug design", "circuit", "molecular machine", "power stroke", "control system" etc. are routinely used by researchers as means to conceptualize and communicate novel biological phenomena encountered at the molecular and cellular scales in terms of our familiar scale of physical reality. However, as has been argued elsewhere, the intuitive appeal and convenience of mechanistic metaphors and interpretations lock the biological thought in the box of inadequate conceptualization, impairing the ability of researchers to understand and model biology (Kurakin, 2004; Kurakin, 2005c; Van Regenmortel, 2007). 
The conventional representations of cellular regulatory and metabolic systems in the form of engineering-like diagrams invariably convey the comforting impression of understanding and control over the corresponding phenomena (Fig. 1). However, to be useful in the same sense as are the design charts of mechanical and electrical engineering, the diagrams of signal transduction and metabolic pathways should necessarily assume exquisite specificity of molecular interactions mediating the intracellular fluxes of energy, matter, and information shown as links in the pathway diagrams. Even though such assumption may seem quite sensible within the clockwork image of the cell and the lock-and-key paradigm of biomolecular recognition, it is in sharp contradiction with experimental reality. A large body of experimental evidence indicates that exquisite specificity is rare in the interactions involving proteins, while ambiguity and adaptive plasticity appear to be a common feature of protein-mediated interactions and chemical conversions.
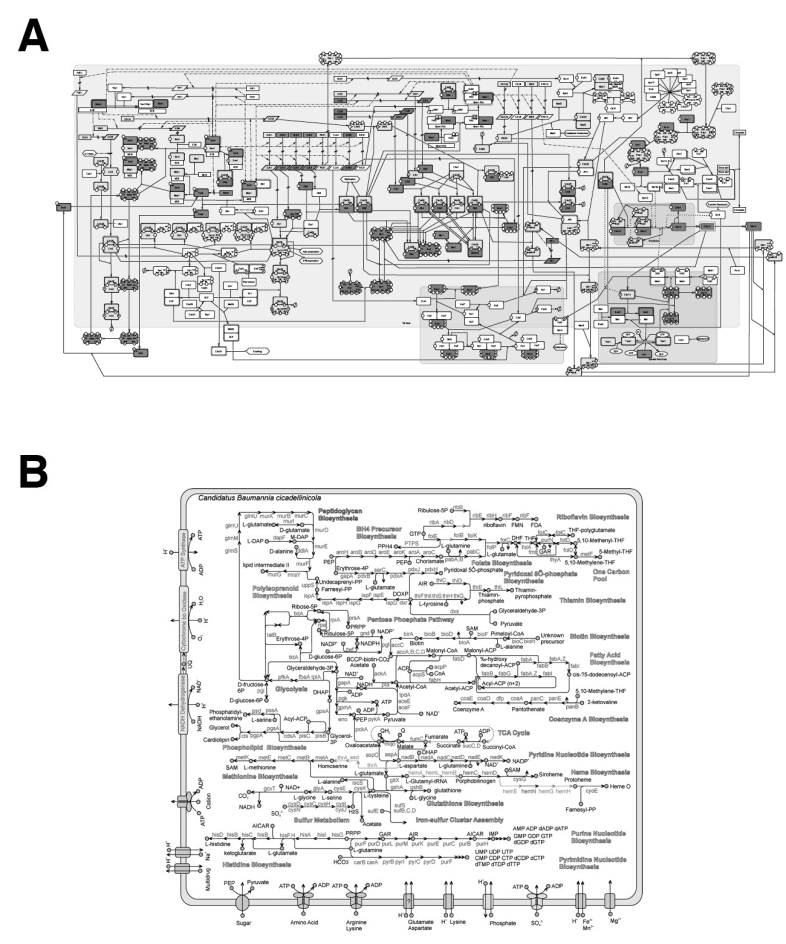

Figure 1. Design charts of cellular circuitry.

Albeit reassuring and intuitively appealing, the concept of design implicit in the conventional charts of metabolic conversions and protein interactions is a misleading and inadequate representation of experimental reality. The inherent ambiguity, probabilistic nature, and context-dependency of links are ignored in such diagrams. As a consequence, the mechanistic assumptions and inferences implied in or derived from biological design diagrams misconstrue reality, thus preventing understanding and adequate modeling of the corresponding phenomena. A) Map of molecular interactions in the cell cycle in S. cerevisiae - reproduced from Figure S4 in Moriya et al. (Moriya et al., 2006). B) Predicted metabolic pathways in $B$. cicadellinicola - reproduced from Figure 4 in $\mathrm{Wu}$ et al. (Wu et al., 2006).

As argued in this article, the contradiction between the inherent ambiguity of molecular recognition and the apparent specificity and order observed in intracellular signaling and metabolic conversions can be resolved by reconceptualizing molecular recognition and cellular organization within the interpretational framework of selforganization. The conceptualization of biological phenomena in terms of self-organization and stochasticity was recently invoked to rationalize a variety of seemingly paradoxical experimental observations pertaining to molecular motors and protein translocation (Kurakin, 2006), sub-cellular organization (Kurakin, 2005a; Misteli, 2001), stochasticity in gene expression, cell plasticity, organism development, and other biological phenomena (Theise, 2005; Theise and Harris, 2006; Kurakin, 2004, 2005b). Introducing and defining such concepts as evolutionary memory, bounded stochasticity, and adaptive plasticity, the present work expands and refines the conceptual framework of self-organization. Unlike mechanistic interpretations, the emerging concepts of selforganization appear to be universal and scale-invariant, holding a promise to unify organizational phenomena across diverse scales of biological organization, from molecules to societies.

\section{Promiscuity of metabolic enzymes}

To explain the emergence of novel metabolic functions in the course of biological evolution without invoking the questionable assumptions required by the textbook model of retrograde evolution (Horowitz, 1945), such as availability and stability of intermediates of the backwardly evolving metabolic pathway, Jensen proposed the recruitment model of metabolism in 1976 (Jensen, 1976). He suggested that the primitive cells possessed a limited amount of genetic information and produced a small number of largely unregulated and substratepromiscuous enzymes-generalists, while the primitive metabolic systems existed as inefficient and probabilistic networks of interdependent chemical reactions that included both enzyme-mediated and non-enzymatic chemical transformations. Jensen also hypothesized that a fortuitous emergence of new metabolites conferring selective advantage to the host cell would drive the 


\section{MS v.03Aweb_SOvsW: Mol. Recognition}

recruitment of enzymes to the pathways generating useful products. In this way, enzymes-generalists were recruited to improve on the already pre-existing but slow and inefficient pathways, rather than creating them de novo. A genetic duplication-divergence mechanism was proposed to be responsible for the emergence of selective and regulated enzymes-specialists under the pressure for metabolic efficiency. It may be argued that the recruitment model "lost" to the textbook variant, despite the apparently uncertain assumptions of the latter (Hartman, 1975; Jensen, 1976), largely because the recruitment model was poorly compatible with the determinism of the clockwork image of the cell and required a radical departure from the dominating "lock-and-key" paradigm of enzyme specificity, while the textbook model was significantly more consistent with conventional mechanistic interpretations. In any case, it appears that experimental data suggesting substrate ambiguity of metabolic enzymes were broadly available and common enough to justify the proposal of the recruitment model already in the 1970s. Many examples are indeed presented and discussed in Jensen's original publication (Jensen, 1976).

An impressive amount of experimental evidence collected since the introduction of the recruitment model indicates that, in addition to substrate ambiguity, catalytic promiscuity is likely to be another common property of metabolic enzymes (Babady et al., 2007; Copley, 2003; James and Tawfik, 2001; O'Brien and Herschlag, 1999). Consequently, O'Brien and Herschlag in their review refined and extended the recruitment model, discussing the ubiquity, possible sources, and advantages of catalytic promiscuity (O'Brien and Herschlag, 1999). The current views on global organization of metabolism together with a recent proposal to treat metabolism in probabilistic terms support Jensen's earlier hypothesis (Nicholson and Wilson, 2003). The modern analytical technologies based on mass spectrometry are revealing the unexpected richness and diversity of metabolites and, by inference, of biotransformations maintained within any given organism (Major et al., 2003). It is proposed that the production of major metabolites simply reflects a high probability of the corresponding conversions, while multiple and diverse micrometabolites are generated by low-probability background biotransformations. "If a metabolite is capable of being produced at all, it will be: it is merely the quantity that is in question." (Nicholson and Wilson, 2003).

"Although enzyme specificity has long been considered the hallmark of biological processes, evidence presented in the past few years indicates that the possibility of protein promiscuity may have been seriously overlooked, simply because it was never routinely
Alexei Kurakin Novato, CA 07/07/2007

addressed" (Macchiarulo et al., 2004). Macchiarulo et al. applied a docking algorithm in the all-against-all computational experiments to cross-dock 125 structures of endogenous $E$. coli metabolites versus 120 structures of metabolic enzymes with the purpose to evaluate in a systematic manner the extent of possible promiscuity in substrate-enzyme interactions. The results of this computational study show that the cognate enzyme-ligand pairs only rarely constitute the best fit, overall revealing a great potential for cross-reactivity in metabolic reactions. Significantly, the authors demonstrate that the highest degree of selectivity is achieved by combining both the selectivity of individual enzymes toward different substrates and the selectivity of individual substrates toward different enzymes, thus suggesting that the specificity of metabolic conversions in the conditions of mutual competition of substrates for enzymes and enzymes for substrates resides, in part, in the matching spectra of metabolites and metabolic enzymes maintained in the cell (Macchiarulo et al., 2004).

\section{Biophysical basis of protein promiscuity}

The progress in research technology and methods together with the advances in our understanding of protein biophysics are bringing about a novel image of the protein emerging as a dynamic and adaptive molecular organization akin to complex adaptive systems (Fenimore et al., 2002; Frauenfelder, 2002; Kurakin et al., 2007; Ma et al., 2002; Van Regenmortel, 2002). In contrast to the textbook images and the conventional computer models representing proteins as rigid Lego block - like structures, the experimental reality suggests that proteins as a class cover an enormous range of structural plasticity, with natively unfolded proteins being one of the extreme ends (Tompa et al., 2005; Uversky, 2002). The opposite end, i.e. the absolutely rigid crystal-like protein structure, is a highly unlikely inhabitant of natural environments. Notably, even the enzymes of extreme thermophiles, which have been stabilized by evolution to withstand denaturing temperatures of hot environments, still require and rely on structural flexibility and dynamics to perform their functions (Berezovsky and Shakhnovich, 2005; Nobe et al., 1998). Combining nuclear magnetic resonance spectroscopy and molecular dynamics simulations Lindorff-Larsen et al. showed that even the hydrophobic cores of tightly folded proteins behave more like liquids rather than solids (Lindorff-Larsen et al., 2005). Single molecule studies, including single molecule enzymology, necessitated the introduction of such notions as static and dynamic disorders, the former to reflect the fact that any population of seemingly identical (isogenic) protein 


\section{MS v.03Aweb_SOvsW: Mol. Recognition}

Alexei Kurakin Novato, CA 07/07/2007 molecules is always composed of different individuals and the latter to indicate the fact that the properties of the same individual molecule change in time (Bai et al., 1999; Xie and Lu, 1999). Re-evaluating the conventional image of molecular recognition, Van Regenmortel pointed out that the binding site is not a given static entity existing without a binding partner, and thus is not defined in the absence of the latter (Van Regenmortel, 2002, 2007). Nussinov et al. argue that any given protein structure exists in solution as a spectrum of multiple conformers that continuously interconvert on different time scales. Because different conformers may potentially accommodate different ligands, the commonly observed promiscuity in protein recognition phenomena becomes a matter of dynamics and re-distribution of the pre-existing conformer populations (Ma et al., 2002). The notion of the protein structure maintained as a bounded set of interconverting conformers is supported by measurements of protein structure dynamics performed on single enzyme molecules (Yang et al., 2003).

Since active sites of enzymes represent highly reactive environments packed with nucleophiles, electrophiles, acids, bases, metal ions, and cofactors, and because the same functional groups can play different roles in different enzymatic reactions (Copley, 2003; O'Brien and Herschlag, 1999), both catalytic promiscuity and substrate ambiguity are only to be expected within the framework of dynamic and adaptive protein structure. They remain surprising and paradoxical only within the "lock-and-key" paradigm of molecular recognition. It is fair to suggest that different enzymes are likely to exhibit different degrees of ambiguity in respect to their substrates and to the types of reactions they catalyze, thus occupying different positions in a wide spectrum of specificity extending from the rigid order of exquisite selectivity to the chaos of unspecific promiscuity.

\section{Specificity and promiscuity in signal transduction}

Whether pictured as a linear pathway or a diagram of cross-talking pathways, the specific protein interactions that mediate intracellular signaling are tacitly assumed to be largely unique and mutually exclusive. A closer and unbiased look at the data reveals, however, that the assumption of exquisite selectivity in specific proteinprotein associations is inconsistent with the results of biochemical studies addressing specificity of protein interactions and fits poorly the current image of dynamic and flexible protein organization, not mentioning the discovery of natively unfolded proteins, many of which are key signaling molecules (Uversky et al., 2005). As an illustrative example, let us consider in more detail perhaps the most studied and well-understood class of specific protein interactions, namely the interactions mediated by signaling protein domains.

Protein interaction modules, such as PDZ, SH3, WW, $\mathrm{EH}, \mathrm{SH} 2$ and other signaling domains, mediate proteinprotein interactions by recognizing and binding short and usually linear peptide epitopes within their interacting partners (Kay et al., 2000; Sudol, 1998). Protein interaction domains (PIDs) constitute structurally and functionally independent modules in the context of their host proteins. They are often found in multiple copies and in different combinations within scaffolding, cytoskeletal, and signaling proteins (Kuriyan and Cowburn, 1997; Pawson, 2007). Speaking of intracellular signaling, protein modules and their selective interactions with peptides have been widely and for a long time perceived as an experimental system that came closest to the "lock-andkey" ideal of protein recognition, (Das and Smith, 2000; $\mathrm{Hu}$ et al., 2004) so that even the existence of a "protein recognition code" governing PID-mediated interactions was prophesized (Sudol, 1998).

Having reviewed available research data, Ladbury and Arold came, however, to the unexpected conclusion that the degree of selectivity provided by $\mathrm{SH} 3$ and $\mathrm{SH} 2$ domains is incommensurate with the specificity observed in the signal transduction events they mediate (Ladbury and Arold, 2000). Their analysis and conclusion challenge the common belief that the specificity in the signal transduction pathways employing $\mathrm{SH} 3$ and $\mathrm{SH} 2$ domains relies exclusively on the molecular recognition selectivity provided by these two domain families (Ladbury and Arold, 2000). Tong et al. applied an approach combining two orthogonal techniques, yeast-two hybrid and phage display, to elucidate interactions of all yeast SH3 domains on the whole proteome scale (Tong et al., 2002). Later on, the Italian team that led the phage display part of the effort, while discussing the problem of insufficient inherent specificity of SH3 domains, commented: "One of the conclusions of this approach is that the inferred number of ligands for each SH3 domain is larger than anticipated. This finding is somewhat disturbing since it is difficult to reconcile with the view that specific cell responses are the result of the precise wiring of the proteins in the cell." (Castagnoli et al., 2004) As an example, Fig. 2 provides a visual illustration of the promiscuity observed among yeast SH3 domains belonging to different specificity classes. In the study by $\mathrm{Hu}$ et al., the 1930 proteomic fragments predicted to interact with WW domains were tested for binding to 57 human WW domains in all-against-all experiments. Most of the WW domains tested bound multiple proteomic 
ligands, somewhere from 53 to 264 ligands each, depending on the identity of individual domains. An "average" WW domain bound to approximately 105 different proteomic fragments with moderate affinity and interacted very strongly with about 39 distinct proteomic ligands (Hu et al., 2004). The notorious promiscuity of PDZ domains is reflected in the continual but so far failed attempts to classify PDZ domains in accord with their specificities (see Refs (Bezprozvanny and Maximov, 2001; Dev, 2004; Harris and Lim, 2001; Hung and Sheng, 2002; Vaccaro and Dente, 2002) for at least five different classifications). Even the most comprehensive tour de force biochemical studies combining the systematic amino acid substitutions of each position in cognate ligands with the accurate $K_{D}$ measurements failed to generate any simple (or complex) rules either for PDZ or for BIR domain recognition (Kipp et al., 2002; Lim et al., 2002).

To summarize, the promiscuity of signaling protein domains has been a subject of multiple experimental studies, reviews, and discussions and can be regarded today as an established experimental fact (Castagnoli $e t$ al., 2004; Keskin and Nussinov, 2007; Kipp et al., 2002; Kurakin et al., 2007; Landgraf et al., 2004; Lim et al., 2002; Ma et al., 2002; Mayer, 2001). However, no satisfying explanations of such promiscuity that would be consistent with conventional deterministic views on cellular organization have been yet proposed.

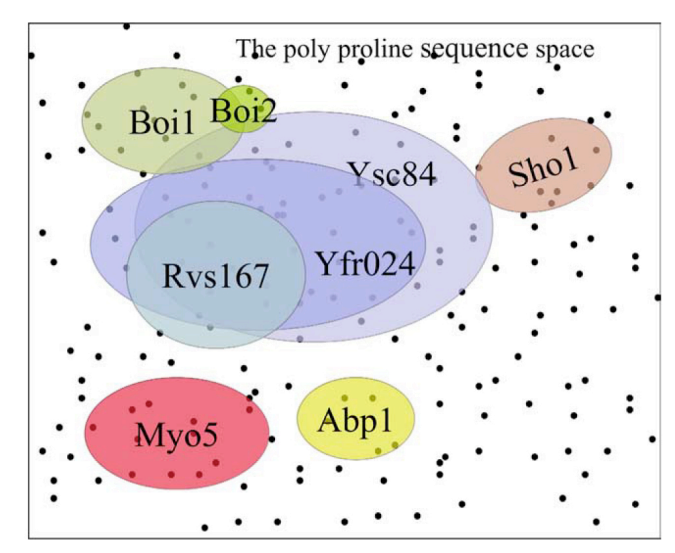

Figure 2. Promiscuity of SH3 domain interactions.

A schematic illustration of the promiscuity observed among the eight yeast $\mathrm{SH} 3$ domains chosen from five different specificity classes (Landgraf et al., 2004). Different peptide sequences are represented as dots in a poly-proline peptide sequence space. The largely overlapping specificities of individual yeast $\mathrm{SH} 3$ domains are shown as colored ovals. It is worth noting that the real extent of promiscuity is underestimated in this illustration, for only the peptides with affinities stronger than an arbitrary $K_{D}$ cutoff of $50 \mu \mathrm{M}$ are included in the ovals.
Reproduced from Castagnoli et al. (Castagnoli et al., 2004) with the kind permission from Federation of the European Biochemical Societies. The data used to generate the schematics are from Langraft et al. (Landgraf et al., 2004).

\section{A study of specificity and adaptive plasticity in PDZ domain recognition}

In order to address in a systematic manner the relationship between specificity and promiscuity commonly observed in the molecular interactions mediated by PDZ domains, we applied recently an array of novel biochemical and statistical approaches to generate and to analyze two large populations of artificial and natural peptide ligands of six PDZ domains from the synaptic proteins PSD95 and SAP97 (Kurakin et al., 2007). Postsynaptic density protein 95 (PSD95) is an archetypal member of the synapse-associated protein (SAP) family of scaffolding molecules that control synaptic composition, organization, and function through the protein interactions mediated by its multiple protein interaction domains (Fujita and Kurachi, 2000; Kim and Sheng, 2004). The SAP proteins share the same overall domain organization with three N-terminal PDZ domains followed by an SH3 domain and a guanylate homology domain at the Cterminus (Fig. 3). Ninety five artificial PDZ domain ligands were isolated from phage-displayed combinatorial peptide libraries and 126 natural ligands were synthesized as 15 -mer proteomic fragments. Both artificial and natural ligands were tested for binding to the target PDZ domains in the all-against-all in vitro binding experiments. The following findings and conclusions of this study are especially relevant for the present discussion.

First, even though these well-studied PDZ domains have been known to recognize and bind short $\mathrm{C}$-terminal sequences sharing $X-(T / S)-X-V-C O O H$ consensus (Hung and Sheng, 2002; Lim et al., 2002), it appears that at least eight C-terminal residues of peptide ligands contribute to the affinity and specificity of the interaction. The remarkable adaptive plasticity and complexity of PDZ domain recognition observed in this and in previous studies appears to reside in the integral nature of the individual ligand residue contributions. Sub-optimal amino acids at some of the ligand positions can be compensated by optimal amino acids at other positions to preserve the overall strength of interaction. At the same time, even the major favorable energetic contributions of threonine and valine within the known consensus $X-(T / S)-X-V-C O O H$ can be compromised by delinquent residues acting somewhere else along the ligand chain. It is worth pointing 
out that such binding behavior is well consistent with an image of dynamic protein structure maintained in solution as differentially populated families of interconverting conformers able to accommodate different ligands (Ma et $a l ., 2002)$. It is also consistent with the idea advanced by Van Regenmortel that the mutual structural co-adaptation of a protein and its ligand, which takes place upon binding, may be a rule rather than an exception in the realm of proteins (Van Regenmortel, 2002, 2007). In other words, adaptive plasticity may represent a general feature of all protein recognition phenomena.

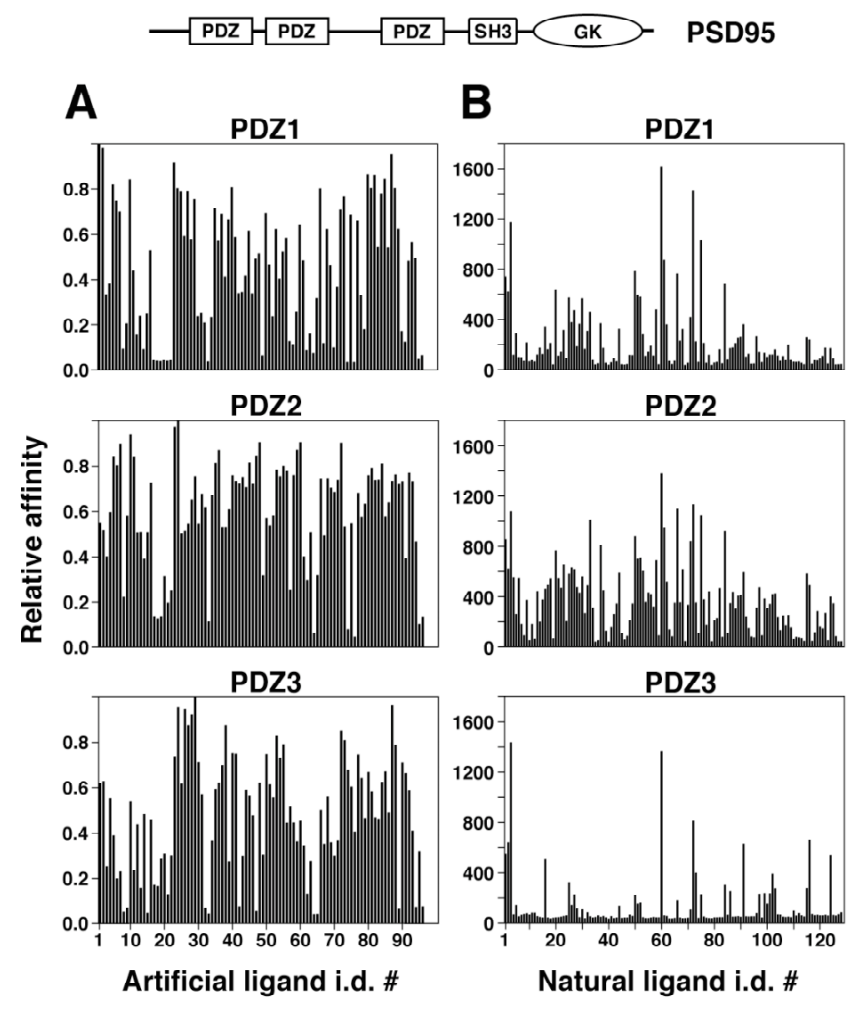

Figure 3. Differential selectivity of the third PSD95 PDZ domain towards artificial and natural ligands.

The relative affinities of the PSD95 PDZ domains towards their cognate artificial ( $A$, normalized) and natural $(B$, arbitrary units) ligands are shown as binding histograms. The domain organization of the PSD95 protein is indicated above the histograms. Ninety five artificial ligands were selected from phage-displayed combinatorial peptide libraries, while the 126 proteomic C-terminal fragments matching the last four residues of artificial ligands were synthesized as 15 -mer peptides. Notice that the third PDZ domain is remarkably selective in its interactions with proteomic fragments but is as promiscuous as the other two domains towards artificial ligands. The comparative analysis of the amino acid compositions of artificial and natural ligands combined with the binding affinity data suggest that what appears as the exquisite selectivity of the third PDZ domain towards proteomic fragments is most likely not an intrinsic property of the domain itself, but a consequence of a limited number of PDZ3 domain interactors present in the human genome (see Ref. (Kurakin et al., 2007) for details). This conclusion suggests an additional source of selectivity residing in the matching spectra of PDZ domains and their ligands encoded in the genome.

The binding histograms are adapted from Kurakin et al. (Kurakin et al., 2007).

Another unexpected finding of our study was a significant difference in the degree of promiscuity exhibited by the same PDZ domain towards artificial as compared to natural ligands. The third PDZ domain of PSD95 (and of SAP97) in our experiments was as promiscuous as the other two PSD95 PDZ domains towards artificial ligands. However, unlike the first two domains, which preserved their largely promiscuous binding behavior toward the peptide ligands derived from the human proteome, the third domain had remarkably few interacting partners among proteomic fragments (Fig. 3). A comparative analysis of the artificial and natural ligands of the third PDZ domain suggested that what appears as an exquisite selectivity towards natural ligands may in fact reflect the selective pressures imposed by evolution on the functional organization of the postsynaptic density, which led to a relatively limited number of PDZ3 domain interactors encoded in the genome. Therefore, it is likely that the selectivity of the intracellular interactions mediated by the PSD95 PDZ domains resides not only in the physico-chemistry of PDZ domains but also in the matching spectra of PDZ domains and their cognate ligands encoded in the genome. It is hardly coincidental that the study by Macchiarulo and colleagues, where large populations of metabolites and metabolic enzymes were cross-docked in all-against-all computational experiments, came independently to essentially the same conclusion, pointing out at the matching spectra of metabolites and metabolic enzymes as an additional source of selectivity in metabolic conversions (Macchiarulo et al., 2004).

\section{The concept of evolutionary memory}

To explain the biological meaning of the selectivity residing in the matching spectra of the SAP PDZ domains and their cognate C-terminal ligands we advanced the hypothesis that the synaptic environmental and organizational invariants are encoded in the genome in the form of such matching spectra. In this way, the genome loosely specifies the overall schematics and principles of synaptic molecular organization, while maturation, finetuning, and adaptation of individual synaptic structures take place as a result of the unique developmental 


\section{MS v.03Aweb_SOvsW: Mol. Recognition}

experiences specific for individual synapses. In the same sense as neuronal organization of every newborn brain has been shaped by evolution to recognize certain perceptual and environmental invariants, but is not limited to recognition of those patterns only, the PDZ domains have been shaped by evolution to recognize certain C-terminal sequences present in a given proteome, but are not limited to the recognition of those sequences only. In this way, the composition, organization, and functioning of individual synapses remain open for evolution at both ontogenetic and phylogenetic time scales, accommodating novel $\mathrm{C}$ terminal sequences that may potentially arise from a plethora of epigenetic and genetic molecular mechanisms known to generate molecular diversities, which include posttranslational modifications, regulated proteolysis, RNA splicing, mutations, DNA rearrangements, protein splicing, and others. We therefore proposed that the adaptive plasticity of PDZ domain recognition and the wide affinity range of the SAP PDZ domain interactions are evolutionarily enforced by the requirement for synapse plasticity and reflect the managerial role of SAP scaffolds within synaptic molecular organizations (Kurakin et al., 2007).

It is not difficult to see how the same conceptualization and logic can be applied to other protein interaction modules, proteins, metabolic enzymes and other biological macromolecules and their interactions, thus giving a fresh perspective on biological organization in general. It is reasonable to hypothesize, for example, that the spectrum of endogenous metabolites within a given organism has its match in the spectrum of metabolic enzymes encoded in the organism's genome. "In truly endogenous processes, the primary determinants of the biochemical transformations are under the direct control of enzymes and transporters of the host genome and have been optimized by evolution." (Nicholson and Wilson, 2003) However, since what is "endogenous" and what is "xenobiotic" are only ends of a continuum, which includes, for example, "sym-endogenous" and "symxenobiotic" metabolites, and since any metabolic organization is not a frozen construct or a machine (then it is dead), but is kept alive, i.e. open to evolution and adaptation on multiple time scales, the substrate ambiguity and catalytic promiscuity of metabolic enzymes are likely to be highly advantageous for the metabolic adaptability of the cell/organism as a whole and, therefore, should be enforced by evolutionary selection. On the other hand, the pressure for metabolic efficiency precludes total promiscuity and is responsible for the continuous maintenance and improvement of the match between current endogenous metabolites and metabolic enzymes.
Alexei Kurakin Novato, CA 07/07/2007

This match outlines, in general terms, what is expected to be the most optimal structure of chemical transformations and energy/matter fluxes for a given organism under given environmental circumstances. Conceptually, it is a guess the organism bets on in the evolutionary game of competition and survival. Because too much structure compromises metabolic adaptability and too much promiscuity compromises metabolic efficiency, the balance between adaptability and efficiency is achieved by producing adaptive enzymes whose individual structures have been optimized by evolution to recognize the invariant or slowly changing features within the landscape of the endogenous metabolome, in exactly the same sense as the neuronal organization of every newborn brain has been shaped by evolution to recognize certain perceptual and environmental invariants of the native habitat. It seems only sensible to call such approach to information storage and retrieval the "evolutionary memory". Unlike design, evolutionary memory, in the same way as the "real" memory studied in neuropsychology (Schacter, 2001), is contextual and flexible. It does rely on the past, but accommodates to the present and remains open to alternative futures.

The concept of evolutionary memory can be conveniently illustrated using a social metaphor of a corporate employee party. Normally, during such parties, even though there is a great deal of commotion and stochasticity, people naturally tend to engage in familiar interactions, unconsciously forming groups and/or tables that reflect their business relationships within the organization. If a party is not too big and not too wild and people are allowed to freely choose their interactors, an attentive observer can usually discern a general structure of organization, albeit in a vague form. The memory of the organizational structure is reflected in the spatiotemporal distribution of interpersonal interactions during an employee party. In the same way, the cellular components - when isolated from their natural cellular environment and tested in the all-against-all interaction experiments in vitro or in silico - will display a certain, albeit limited, selectivity as a memory of the familiar interactions that took place so often in the evolutionary past.

An employee party is not, however, the most efficient way to conduct business. What makes a business organization efficient is its internal spatio-temporal organization and orchestration of interpersonal interactions and transactions during business hours, which is achieved through the microcompartmentation of individual activities in space and time, the channeling of energy/matter/information fluxes and through the organization and maintenance of regulated business 


\section{MS v.03Aweb_SOvsW: Mol. Recognition}

routines. It is reasonable to suggest that the muchadvocated intracellular organization (Clegg and Wheatley, 1991; Hochachka, 1999; Masters, 1991; Ovadi et al., 2004; Porter, 1984; Welch and Clegg, 1987), which is based on the same principles of channeling, dynamic microcompartmentation, and regulation, and which is routinely destroyed during conventional isolation procedures, is that what makes the intracellular fluxes of energy/matter/information so apparently specific and efficient and the performance of the cell competitive, even though its individual constituents exhibit only limited selectivity when isolated from their cognate organizational context.

\section{Inter-scale conservation of adaptive plasticity}

It is generally accepted that the adaptability of human societies and organizations depends and relies on the plasticity of the human brain able to learn from the past, adapt to the present and foresee the future. The plasticity of the human brain depends and relies on the plasticity of the synaptic connections defining the organizational structure of neuronal networks in the brain. In its turn, the synaptic plasticity underlying memory and learning depends and relies on the plasticity of the signalprocessing molecular organizations that constitute the postsynaptic density (PSD). It is only logical to suggest that the plasticity of the PSD molecular organization necessarily requires and relies on the plasticity of the protein-protein interactions defining the organizational structure and dynamics of PSD. Being central organizers of the postsynaptic density, synapse-associated protein scaffolds such as PSD95, SAP97, and others define, in significant measure through their PDZ domain-mediated associations, the composition, spatial organization, and spatiotemporal dynamics of PSD. The adaptive plasticity of the PDZ domain and the adaptive plasticity of human society are thus intimately linked through the conservation of adaptive plasticity across different scales of biological organization (Fig. 4).

Competition within PSD between available C-termini of channels, receptors, and other protein molecules for available PDZ domains of scaffolds, competition within the brain between available dendritic spines for available axonal terminals, competition within the business organization between available employees and recruits for available positions and competition within the free market economy between available firms for available market opportunities constitute together a hierarchically-organized nested set of the selective pressures that enforce adaptive plasticity at different spatio-temporal scales simultaneously and interdependently. Locking-in on the
Alexei Kurakin Novato, CA 07/07/2007

same interactions at the scale of PDZ domains in PSD would compromise synaptic plasticity, thus locking-in and compromising the performance and adaptability of the affected human brain and as a consequence compromising the performance and adaptability of the business organization this human brain is a part of, especially if it happens to be the brain of a senior manager. It is not difficult to see how the same logic applies to any biological system surviving or competing under the pressure of evolutionary selection. Since "nothing in biology makes sense except in the light of evolution" (Dobzhansky, 1973), it can be concluded that the interscale conservation of adaptive plasticity is a fundamental and defining property of biological organization. It is reasonable to suggest that adaptive plasticity is achieved at each and every scale of biological organizational hierarchy through what can be called "bounded stochasticity", i. e. through stochastic behavior within the bounds set by evolutionary memory.

\section{Universality of self-organizational concepts and principles}

It appears that the concept of design, when applied to or implied in the diagrams of biomolecular interactions, is inappropriate and misleading, at least because the multiple, and potentially innumerable, open and alternative links that exist or may exist in reality are simply not shown on such diagrams (Fig. 4, inset). The inherently probabilistic nature of links and their dependence on the environmental context are also ignored. Every protein molecule has many choices with respect to its possible interactions and these choices are highly context-dependent. The spectrum of possible choices is likely to be defined by both phylogenetic and ontogenetic evolutionary histories of any given protein molecule. At the very best the diagrams of signal transduction pathways, protein interactions, and metabolic conversions represent snap-shots of "conversations" taking place during a "corporate employee party", to use the above mentioned social metaphor. They indeed contain the information about what are likely to be "business relationships" during "business hours" in the context of native "business organizational structure", but such information is residual, probabilistic in its nature, and is far from being a design chart, in both conceptual and practical terms. It seems appropriate to conceptualize such information as a memory of co-evolutionary relationships and to replace the concept of design by the concept of evolutionary memory.

Stochasticity was suggested recently as a general principle of differentiation and adaptation at all scales of 


\section{Adaptive plasticity and interdependency of organizational hierarchies}

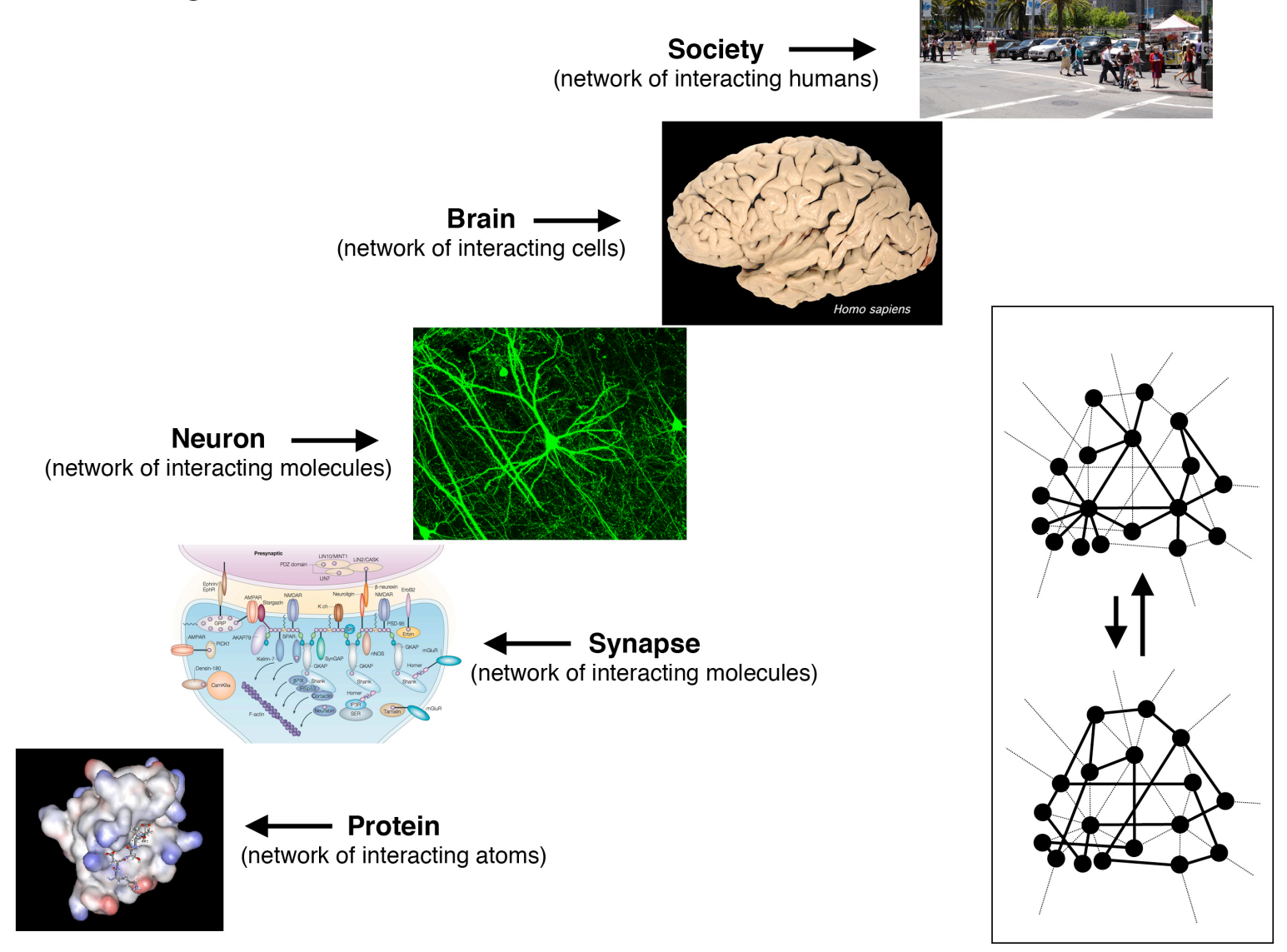

Figure 4. Conservation of adaptive plasticity across scales of biological organization.

Such complex adaptive systems as proteins, cells, organisms, and organizations co-exist and co-evolve at different spatiotemporal scales, forming a nested set of interdependent organizational hierarchies. The adaptive plasticity at any given scale builds upon and requires plasticity of the underlying scale organizations. The evolutionary pressures of competition and selection, which favor adaptability in a continuously changing world, enforce the conservation of adaptive plasticity across all scales of biological organizational hierarchy.

The inset shows a universal conceptualization of complex adaptive systems in terms of networks. Whether we speak of a protein, a sub-cellular structure, a cell, an organism, or an organization, they all can be conceptualized as a metastable network, where links invariably represent exchanges of energy, matter, and/or information between the corresponding constituent parts, be it atoms, molecules, cells, or organisms. The links in such networks are of inherently probabilistic and dynamic nature. The major fluxes of energy, matter, or information of a given network configuration (shown as bold links) are always embedded in a sea of low probability exchanges (shown as dotted links). A switch to an alternative network configuration of a biological system in the course of adaptation and/or evolution necessarily requires and relies on adaptive plasticity of nodes able to find, to establish, and to maintain new links. In reality, for any given network configuration, the strengths of links or the frequencies of exchanges between nodes do not follow simply bimodal distributions - as shown here with bold and dotted links - but are usually described by heavy-tail distributions such as power law or log-normal, as has been demonstrated for such real-world systems as neuronal cortical circuits (Song et al., 2005) as well as metabolic (Almaas et al., 2004; Emmerling et al., 2002) and gene regulation networks (Farkas et al., 2003). Also, real-world networks normally possess multiple metastable configurations characterized by different occupational probabilities, switching between them with different transition probabilities in the process of adaptation.

Sources of the images shown: Society - Union Square, San Francisco; Brain - image of a human brain (credits to Todd Preuss, Yerkes Primate Research Center) is reproduced from Figure 1 in Ref. (Bradbury, 2005); Neuron - the pyramidal neuron "dow" is reproduced from Figure 6(d) in Lee et al. (Lee et al., 2006); Synapse - a schematic diagram of the PDZ protein organization at a mammalian excitatory synapse is reprinted from Kim and Sheng (Kim and Sheng, 2004) with permission from Macmillan Publishers Ltd: Nat. Rev. Neurosc. (C) 2004; Protein - a syntrophin PDZ domain complexed with its peptide ligand (PDB id. 2PDZ) was visualized using the WebLab ${ }^{\mathrm{TM}}$ ViewerLite 3.2 software. Network configurations in the inset were adapted from Jeong et al. (Jeong et al., 2000). 


\section{MS v.03Aweb_SOvsW: Mol. Recognition}

biological organizational complexity (Kurakin, 2005b). Whether we speak about molecules (Xie and Lu, 1999), cells (Hume, 2000; Kurakin, 2005c), or organisms (Fewell, 2003; Herndon et al., 2002), their behaviors are inherently stochastic within the bounds set by their corresponding internal structures. It is reasonable to suggest that their structures, which embody their corresponding evolutionary memories (both ontogenetic and phylogenetic) of previous encounters with their respective environments, are always maintained as a dynamic compromise between economic efficiency and adaptability. Therefore, a more appropriate term for what is perceived within the mechanistic paradigm as promiscuity is adaptive plasticity, which is achieved through stochasticity within the limits set by internal organizational structure.

Adaptive plasticity of higher hierarchies of biological organization builds upon and relies on the adaptive plasticity of underlying hierarchies. Promiscuity of interatomic interactions underlies the adaptability of protein structure. Substrate ambiguity and catalytic promiscuity of metabolic enzymes underlies the adaptability of metabolism (Jensen, 1976; O'Brien and Herschlag, 1999). Promiscuity in protein interactions underlies the adaptability of cellular organization (Kurakin, 2005a; Tompa et al., 2005). Functional promiscuity of moonlighting proteins was proposed to underlie the integration and plasticity of cellular functional systems (Kurakin, 2004, 2005a). Promiscuity of gene expression in differentiated cells may underlie the adaptability of tissue and organism physiology (Kurakin, 2005b). Plasticity of interpersonal relationships underlies the adaptability of social and business networks and organizations, and so forth.

It should be pointed out that unlike the mechanistic interpretations that are fairly well accepted in biology at some spatiotemporal scales but not so well at other scales, the emerging concepts and principles of self-organization are scale-invariant, i.e. they are equally well applicable at different scales of space and time. Such mechanistic concepts as design and determinism, for example, have been actively, and as shown here and elsewhere (Kurakin, 2004; Kurakin, 2005c) erroneously, applied largely at the molecular, sub-cellular, and cellular scales, where the mechanistic interpretations, inferences, and assumptions could survive for a long time without being thoroughly tested due to the absence of adequate technologies. The concept of design has traditionally had a much weaker standing already at the scale of developing organism, and few humans would agree that they are machines and that their behaviors are deterministic. Not design and
Alexei Kurakin Novato, CA 07/07/2007

determinism, but choice and free will are the preferred concepts at the scale of humans and their organizations.

Unlike mechanistic interpretations, self-organization as a concept of emergence, stochasticity as a general principle of differentiation and adaptation, bounded stochasticity as the choices limited by internal organizational structure, evolutionary memory as the biological structure balancing economic efficiency and adaptability, adaptive plasticity and other related concepts and principles constitute together an emerging conceptual framework that is consistent with experimental reality at all scales of biological organization and is universally meaningful whether one speaks about biomolecules, subcellular structures, cells, organisms, or social and business organizations, and whether one considers phylogenetic, ontogenetic, or other time scales. The concepts of stochasticity, adaptive plasticity, and evolutionary memory bring such traditionally human attributes as choice, decision-making, memory and, ultimately, intelligence to the molecular and cellular scales, providing harmonious conceptual continuity across different hierarchical levels of biological organization and, at last, giving justice to the principle of self-consistency. Intelligence begets intelligence. Machines beget only machines.

\section{Concluding remarks}

The present article is a continuation of a series of publications aimed to demonstrate that the mechanistic interpretations, inferences, and assumptions, which have been dominating biological thinking and research for many decades, become today increasingly and massively inconsistent with the experimental reality generated by modern research technologies in many specialized research areas of molecular and cell biology, thus suggesting a global crisis of the mechanistic paradigm in life sciences (Kurakin, 2004, 2005a, b; Kurakin, 2005c; Kurakin, 2006). It should be pointed out that this crisis has been brought about in significant measure by technological progress, and especially so by the computer revolution that allowed for and stimulated rapid advances in analytical and detection technologies and methods. Nanomanipulation, single-molecule studies, fluorescent-based tracers, advanced imaging techniques, analysis of cellular populations on individual cell basis, measurements of molecular dynamics in living cells and organisms, genomic and proteomic technologies, computer analysis and simulation of large-scale experimental data are but a few examples of the technologies and methods that are generating today the experimental reality that challenges the mechanistic conception of living matter. In remarkable 
MS v.03Aweb_SOvsW: Mol. Recognition

correspondence with the scenario of scientific development suggested by Thomas Kuhn in his classic text "The Structure of Scientific Revolutions", the very success of a dominating paradigm brings about its own crisis and necessitates a paradigm shift, as the advances in technology and methods lead to a systematic and widespread accumulation of experimental data that cannot be readily explained and accommodated within the existing conceptual framework (Kuhn, 1996).

If Kuhn's analysis is correct, the awareness of a paradigm crisis is always followed by an exciting phase of extraordinary research and by a splendid array of new scientific discoveries. However, the awareness should come first.

\section{Acknowledgements}

I would like to thank Prof. Marc H. V. Van Regenmortel (Ecole Supérieure de Biotechnologie de Strasbourg) for editing the manuscript and valuable suggestions. I thank Dr. Veronica Galvan (Buck Institute) for critical reading of the manuscript and Dr. Dale E. Bredesen (Buck Institute) for support and encouragement. This work was supported by N545093 and NS33376 grants from the NIH to Dr. D. E. Bredesen.

\section{References}

Almaas E, Kovacs B, Vicsek T, Oltvai ZN, Barabasi AL. 2004. Global organization of metabolic fluxes in the bacterium Escherichia coli. Nature 427: 839-843.

Babady NE, Pang YP, Elpeleg O, Isaya G. 2007. Cryptic proteolytic activity of dihydrolipoamide dehydrogenase. Proc Natl Acad Sci U S A 104: 61586163.

Bai C, Wang C, Xie XS, Wolynes PG. 1999. Single molecule physics and chemistry. Proc Natl Acad Sci U S A 96: 11075-11076.

Berezovsky IN, Shakhnovich EI. 2005. Physics and evolution of thermophilic adaptation. Proc Natl Acad Sci U S A 102: 12742-12747.

Bezprozvanny I, Maximov A. 2001. Classification of PDZ domains. FEBS Lett 509: 457-462.

Bradbury J. 2005. Molecular insights into human brain evolution. PLoS Biol 3: e50.

Castagnoli L, Costantini A, Dall'Armi C, Gonfloni S, Montecchi-Palazzi L, Panni S, Paoluzi S, Santonico E, Cesareni G. 2004. Selectivity and promiscuity in the interaction network mediated by protein recognition modules. FEBS Lett 567: 74-79.
Alexei Kurakin Novato, CA 07/07/2007

Clegg JS, Wheatley DN. 1991. Intracellular Organization: Evolutionary Origins and Possible Consequences to Metabolic Rate Control in Vertebrates. Am. Zool. 31: 504-513.

Copley SD. 2003. Enzymes with extra talents: moonlighting functions and catalytic promiscuity. Curr Opin Chem Biol 7: 265-272.

Das S, Smith TF. 2000. Identifying nature's protein Lego set. Adv Protein Chem 54: 159-183.

Dev KK. 2004. Making protein interactions druggable: targeting PDZ domains. Nat Rev Drug Discov 3: $1047-$ 1056.

Dobzhansky T. 1973. Nothing in Biology Makes Sense Except in the Light of Evolution. The American Biology Teacher 35: 125-129.

Emmerling M, Dauner M, Ponti A, Fiaux J, Hochuli M, Szyperski T, Wuthrich K, Bailey JE, Sauer U. 2002. Metabolic flux responses to pyruvate kinase knockout in Escherichia coli. J Bacteriol 184: 152-164.

Farkas I, Jeong H, Vicsek T, Barabasi A, Oltvai Z. 2003. The topology of the transcription regulatory network in the yeast, Saccharomyces cerevisiae. Physica A 318: 601-612.

Fenimore PW, Frauenfelder H, McMahon BH, Parak FG. 2002. Slaving: solvent fluctuations dominate protein dynamics and functions. Proc Natl Acad Sci U S A 99: 16047-16051.

Fewell JH. 2003. Social insect networks. Science 301: 1867-1870.

Frauenfelder H. 2002. Proteins: paradigms of complexity. Proc Natl Acad Sci U S A 99 Suppl 1: 2479-2480.

Fujita A, Kurachi Y. 2000. SAP family proteins. Biochem Biophys Res Commun 269: 1-6.

Harris BZ, Lim WA. 2001. Mechanism and role of PDZ domains in signaling complex assembly. J Cell Sci 114: 3219-3231.

Hartman H. 1975. Speculations on the origin and evolution of metabolism. J Mol Evol 4: 359-370.

Herndon LA, Schmeissner PJ, Dudaronek JM, Brown PA, Listner KM, Sakano Y, Paupard MC, Hall DH, Driscoll M. 2002. Stochastic and genetic factors influence tissue-specific decline in ageing $\mathrm{C}$. elegans. Nature 419: 808-814.

Hochachka PW. 1999. The metabolic implications of intracellular circulation. Proc Natl Acad Sci U S A 96: 12233-12239.

Horowitz NH. 1945. On the Evolution of Biochemical Syntheses. Proc Natl Acad Sci U S A 31: 153-157.

Hu H, Columbus J, Zhang Y, Wu D, Lian L, Yang S, Goodwin J, Luczak C, Carter M, Chen L, James M, Davis R, Sudol M, Rodwell J, Herrero JJ. 2004. A map 
of WW domain family interactions. Proteomics 4: 643-655.

Hume DA. 2000. Probability in transcriptional regulation and its implications for leukocyte differentiation and inducible gene expression. Blood 96: 2323-2328.

Hung AY, Sheng M. 2002. PDZ domains: structural modules for protein complex assembly. $\mathrm{J}$ Biol Chem 277: 5699-5702.

James LC, Tawfik DS. 2001. Catalytic and binding polyreactivities shared by two unrelated proteins: The potential role of promiscuity in enzyme evolution. Protein Sci 10: 2600-2607.

Jensen RA. 1976. Enzyme recruitment in evolution of new function. Annu Rev Microbiol 30: 409-425.

Jeong H, Tombor B, Albert R, Oltvai ZN, Barabasi AL. 2000. The large-scale organization of metabolic networks. Nature 407: 651-654.

Kay BK, Kasanov J, Knight S, Kurakin A. 2000. Convergent evolution with combinatorial peptides. FEBS Lett 480: 55-62.

Keskin O, Nussinov R. 2007. Similar binding sites and different partners: implications to shared proteins in cellular pathways. Structure 15: 341-354.

Kim E, Sheng M. 2004. PDZ domain proteins of synapses. Nat Rev Neurosci 5: 771-781.

Kipp RA, Case MA, Wist AD, Cresson CM, Carrell M, Griner E, Wiita A, Albiniak PA, Chai J, Shi Y, Semmelhack MF, McLendon GL. 2002. Molecular targeting of inhibitor of apoptosis proteins based on small molecule mimics of natural binding partners. Biochemistry 41: 7344-7349.

Kuhn T. 1996. The Structure of Scientific Revolutions. The University of Chicago Press, Chicago and London.

Kurakin A. 2004. Self-Organization versus Watchmaker: stochasticity and determinism in molecular and cell biology. (http://www.alexeikurakin.org/text/ak030304.pdf)

Kurakin A. 2005a. Self-organization versus Watchmaker: stochastic dynamics of cellular organization. Biol Chem 386: 247-254.

Kurakin A. 2005b. Self-organization vs Watchmaker: stochastic gene expression and cell differentiation. Dev Genes Evol 215: 46-52.

Kurakin A. 2005c. Stochastic cell. IUBMB Life 57: 59-63.

Kurakin A. 2006. Self-organization versus watchmaker: molecular motors and protein translocation. Biosystems 84: 15-23.

Kurakin A, Swistowski A, Wu SC, Bredesen DE. 2007. The PDZ Domain as a Complex Adaptive System. (to be published elsewhere).
Kuriyan J, Cowburn D. 1997. Modular peptide recognition domains in eukaryotic signaling. Annu Rev Biophys Biomol Struct 26: 259-288.

Ladbury JE, Arold S. 2000. Searching for specificity in SH domains. Chem Biol 7: R3-8.

Landgraf C, Panni S, Montecchi-Palazzi L, Castagnoli L, Schneider-Mergener J, Volkmer-Engert R, Cesareni G. 2004. Protein interaction networks by proteome peptide scanning. PLoS Biol 2: E14.

Lee WC, Huang H, Feng G, Sanes JR, Brown EN, So PT, Nedivi E. 2006. Dynamic remodeling of dendritic arbors in GABAergic interneurons of adult visual cortex. PLoS Biol 4: e29.

Lim IA, Hall DD, Hell JW. 2002. Selectivity and promiscuity of the first and second PDZ domains of PSD-95 and synapse-associated protein 102. J Biol Chem 277: 21697-21711.

Lindorff-Larsen K, Best RB, Depristo MA, Dobson CM, Vendruscolo M. 2005. Simultaneous determination of protein structure and dynamics. Nature 433: 128-132.

Ma B, Shatsky M, Wolfson HJ, Nussinov R. 2002. Multiple diverse ligands binding at a single protein site: a matter of pre-existing populations. Protein $\mathrm{Sci}$ 11: 184-197.

Macchiarulo A, Nobeli I, Thornton JM. 2004. Ligand selectivity and competition between enzymes in silico. Nat Biotechnol 22: 1039-1045.

Major H, Castro-Perez J, Nicholson JK, Wilson ID. 2003. Characterisation of putative pentose-containing conjugates as minor metabolites of 4-bromoaniline present in the urine of rats following intraperitoneal administration. Rapid Commun Mass Spectrom 17: 7680.

Masters C. 1991. Cellular differentiation and the microcompartmentation of glycolysis. Mech Ageing Dev 61: 11-22.

Mayer BJ. 2001. SH3 domains: complexity in moderation. J Cell Sci 114: 1253-1263.

Misteli T. 2001. The concept of self-organization in cellular architecture. J Cell Biol 155: 181-185.

Moriya H, Shimizu-Yoshida Y, Kitano H. 2006. In vivo robustness analysis of cell division cycle genes in Saccharomyces cerevisiae. PLoS Genet 2: e111.

Nicholson JK, Wilson ID. 2003. Opinion: understanding 'global' systems biology: metabonomics and the continuum of metabolism. Nat Rev Drug Discov 2: 668-676.

Nobe Y, Kawaguchi S, Ura H, Nakai T, Hirotsu K, Kato R, Kuramitsu S. 1998. The novel substrate recognition mechanism utilized by aspartate aminotransferase of the extreme thermophile Thermus thermophilus HB8. 
J Biol Chem 273: 29554-29564.

O'Brien PJ, Herschlag D. 1999. Catalytic promiscuity and the evolution of new enzymatic activities. Chem Biol 6: R91-R105.

Ovadi J, Orosz F, Hollan S. 2004. Functional aspects of cellular microcompartmentation in the development of neurodegeneration: mutation induced aberrant proteinprotein associations. Mol Cell Biochem 256-257: 8393.

Pawson T. 2007. Dynamic control of signaling by modular adaptor proteins. Curr Opin Cell Biol 19: 112-116.

Porter KR. 1984. The cytomatrix: a short history of its study. J Cell Biol 99: 3s-12s.

Schacter D. 2001. The Seven Sins of Memory: How the Mind Forgets and Remembers. Houghton Mifflin, New York.

Song S, Sjostrom PJ, Reigl M, Nelson S, Chklovskii DB. 2005. Highly nonrandom features of synaptic connectivity in local cortical circuits. PLoS Biol 3: e68.

Sudol M. 1998. From Src Homology domains to other signaling modules: proposal of the 'protein recognition code'. Oncogene 17: 1469-1474.

Theise ND. 2005. Now you see it, now you don't. Nature 435: 1165.

Theise ND, Harris R. 2006. Postmodern biology: (adult) (stem) cells are plastic, stochastic, complex, and uncertain. Handb Exp Pharmacol 174: 389-408.

Tompa P, Szasz C, Buday L. 2005. Structural disorder throws new light on moonlighting. Trends Biochem Sci 30: 484-489.

Tong AH, Drees B, Nardelli G, Bader GD, Brannetti B, Castagnoli L, Evangelista M, Ferracuti S, Nelson B, Paoluzi S, Quondam M, Zucconi A, Hogue CW, Fields S, Boone C, Cesareni G. 2002. A combined experimental and computational strategy to define protein interaction networks for peptide recognition modules. Science 295: 321-324.

Uversky VN. 2002. What does it mean to be natively unfolded? Eur J Biochem 269: 2-12.

Uversky VN, Oldfield CJ, Dunker AK. 2005. Showing your ID: intrinsic disorder as an ID for recognition, regulation and cell signaling. J Mol Recognit 18: 343384.

Vaccaro P, Dente L. 2002. PDZ domains: troubles in classification. FEBS Lett 512: 345-349.

Van Regenmortel MHV. 2002. A paradigm shift is needed in proteomics: 'structure determines function' should be replaced by 'binding determines function'. J Mol
Recognit 15: 349-351.

Van Regenmortel MHV. 2007. The rational design of biological complexity: a deceptive metaphor. Proteomics 7: 965-975.

Welch GR, Clegg JS. (eds) 1987. Organization of Cell Metabolism. Plenum Press, New York.

Wu D, Daugherty SC, Van Aken SE, Pai GH, Watkins KL, Khouri H, Tallon LJ, Zaborsky JM, Dunbar HE, Tran PL, Moran NA, Eisen JA. 2006. Metabolic complementarity and genomics of the dual bacterial symbiosis of sharpshooters. PLoS Biol 4: e188.

Xie XS, Lu HP. 1999. Single-molecule enzymology. J Biol Chem 274: 15967-15970.

Yang H, Luo G, Karnchanaphanurach P, Louie TM, Rech I, Cova S, Xun L, Xie XS. 2003. Protein conformational dynamics probed by single-molecule electron transfer. Science 302: 262-266. 